\title{
Experiments With Tandem Planes
}

\section{The Langley and Eiffel Models, and a New Design}

\section{By Robert Gilbert Ecob}

A GREAT amount of time, energy and money has bee spent on various types of tandem plane machines, an while some success has been attained, the final solution has by no means been reached.

Dr. Langley and M. Eiffel showed very clearly that: 1. In some cases a tandem arrangement of two planes may be more efficient than a biplane arrangement.

2. In a tandem arrangement of two planes it is mor efficient to have both planes at equal angles than it is to have the rear plane at a negative angle.

3. It is more efficient to have a wide than a narrow spacing.

4. Changing the angle of incidence of the rear plane lats a very great vertical steering tendency.

5. A tandem machine will fly.

6. In tandem plane constructions the full size machines follow the same general laws as the models.

It is certain that the 400 h.p. motors in use today woud fly the Langley machine at such a speed that it would be whipped to ribbons by the wind, but it is doubtful if the 50 h.p. motor of Dr. Langley's would be powerful enough to lift a modern biplane as much as a single inch off the ground. If we consider the efficiency of an airplane as so many pounds carried er borse power miles per hour, we conclude that there per horse power miles per hour, we conchde that the ley's interpretation of aerodynamics.

It is easily seen and has been proven that an aerofoil moving through air produces various eddies and cur. rents in its wake. At flying angles the eddies are much reduced, but a decided downward trend is produced. Air, being an elastic medium, tends to return to a state of calm after the passage of the aerofoil. As a generaltheory the down close hind the trailing edge increases. We do not know how soon or at what distance, either relative or absolute, the air will become calm after the passage of the aerofoil, but we do know that there must be some point at which the air will show no appreciable effect from the passage of the aerofoil. Some people claim that the downwash is changed to an upwash, but this is by no down is ch tried in the

Fig. 2 illustrates the flow of air around an aerofoil at a flying angle. No data is available on the condition of the air at a greater distance behind the aerofoil, but it is safe to assume that the downwash will dimin ish rather than be augmented. The following plane is shown in the same relative position as the followin

In all experiments with varying horizontal separations of planes, varying from 2 to 5 chord lengths, the
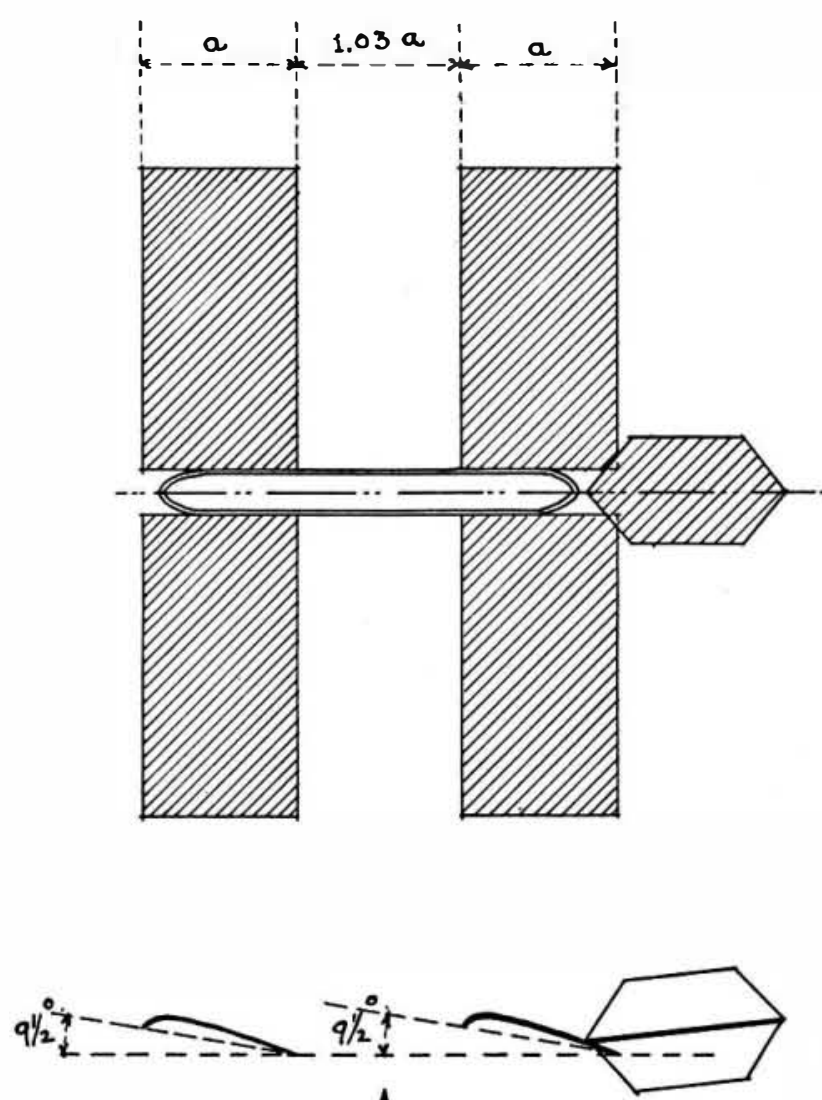

A
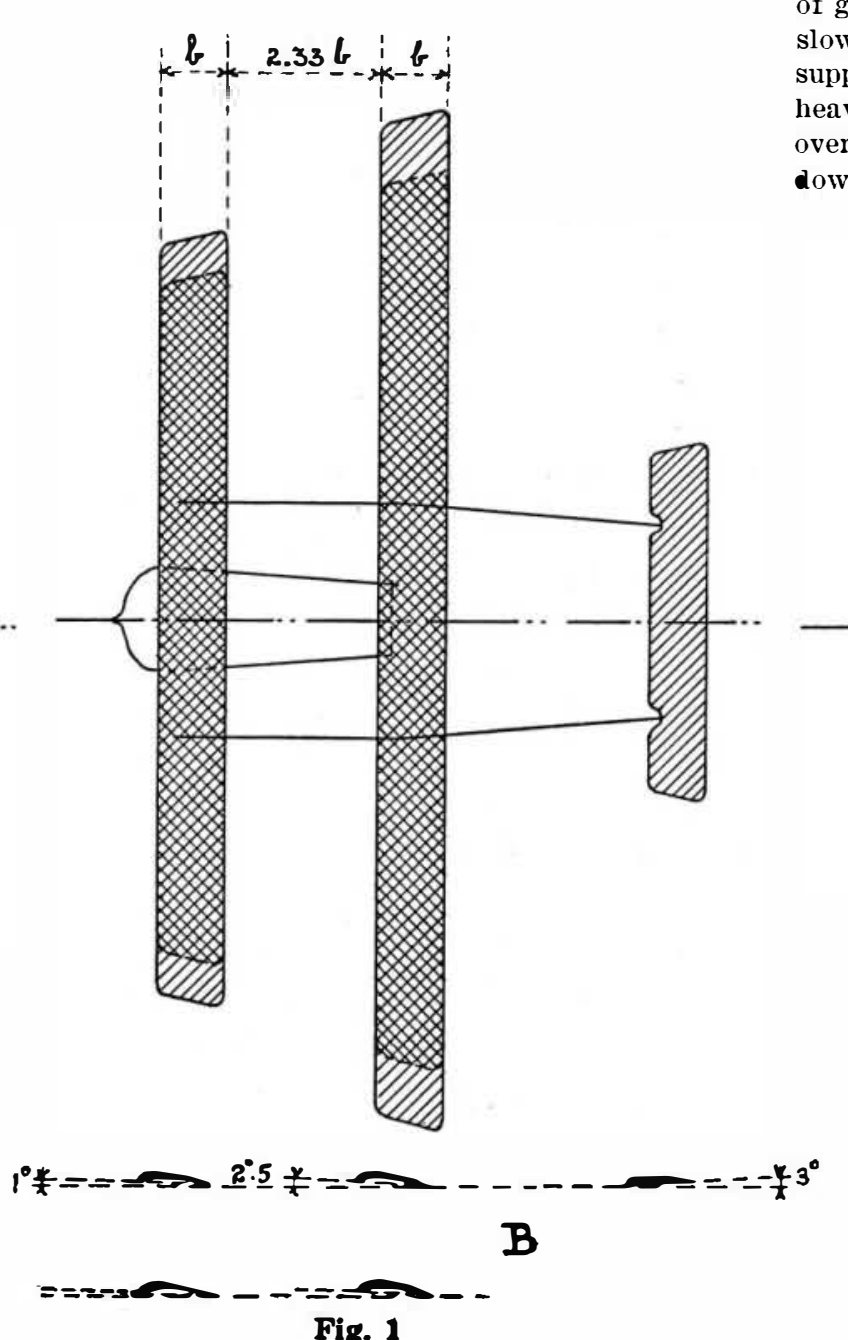

with six planes was studied more carefully, not because it promised the best results, but because it seemed best adapted to full size machines.

The model shown in the photograph on this page is one of a series, the weights of which varied from $11 \frac{1 / 2}{2}$ oz. to $14 \mathrm{oz}$. The wing area is $350 \mathrm{sq}$. inches. It is evident that the planes are lightly loaded.

The models were built to make a rough estimate of the function of the following planes. The plane units or cells were fitted loosely on the bodies and could be shifted fore and aft very easily, as they were held down only by rubber bands.

The location of the centre of gravity relative to the centre of pressure formed a good index to the amount of work performed by the following planes. The centre of gravity could not well be shifted without changing the weight of the model, but by shifting the planes the location of the centre of pressure could be changed at will, without altering the model in other respects, and consequently various changes could be studied closely, and the results compared by the actual performance of the models in free llight.

Referring to Fig. 3, if there were no interference of planes the centre of gravity should be located at $\mathrm{C}_{1} \mathrm{G}_{1}$ to secure horizontal flight. As a matter of fact the models did fly with this arrangement, but they were too tail heavy, and were greatly improved by locating the centre of gravity at CG.

A model of the plane shown in Fig. 3

tive longitudinal diedral angle, and was said to be very stable.

Fig. 1 shows Dr. Langley's tandem monoplane at $\mathbf{A}$ the Jeansen-Calliex tandem biplane at $\mathbf{B}$, and the sixplane tandem models at $\mathrm{C}$. It should be noted that the planes in arrangement $\mathbf{A}$ are very wide from front to back, they are heavily cambered, spaced very close together, and set at greater angles than are ever used in modern practice. The tail must have caused considerable loss of efficiency.

In arrangement $\mathbf{B}$ the planes are spaced farther apart and the rear planes are larger than the front planes. The longitudinal diedral is produced by setting the tail at a negative angle, but as it is spaced very far aft the steering effect on the whole machine would be very great, but the loss from having a negative surface would have to be made up by having a greater area of positive surface.

Arrangement $\mathrm{C}$ was very satisfactory and was achieved after numerous trials with models having four, five, six, eight, and ten planes. The arrangement A very small change in the spacing of the planes relative to the centre of gravity produced a great difference in the performance of the models. A shift of one-eighth of an inch in the two forward units made no perceptible change in the location of the centre of gravity, but in flight it made the difference of the model t the model to a height of 20 feet or causing it to barely
rise higher than six inches from the floor.

or the intermediate planes of the rear planes were doing the lifting, the top intermediate plane was removed. Greater speed was produced, as might be expected owing to decreased head resistance. The model flew very low, probabiy due to the fact that the surface had been decreased. The balance seemed to be the same or so nearly the The bat the results could not be noticel. The other internediate plane was next removed had been so much reduced that the model could not fly. In straightaway flight the models performed very uniformly and steadily. It was noted that toward the end of a flight, as the power died out, every model had a tendency to rise, which might have been due to the fact that the centre of thrust was above the centre of gravity, or it might have been that as the propellers slowed down, the from them did not continuers slowe to heavy however, so that the rising tendency was soon overcome by the force of gravity pulling the noses downward.

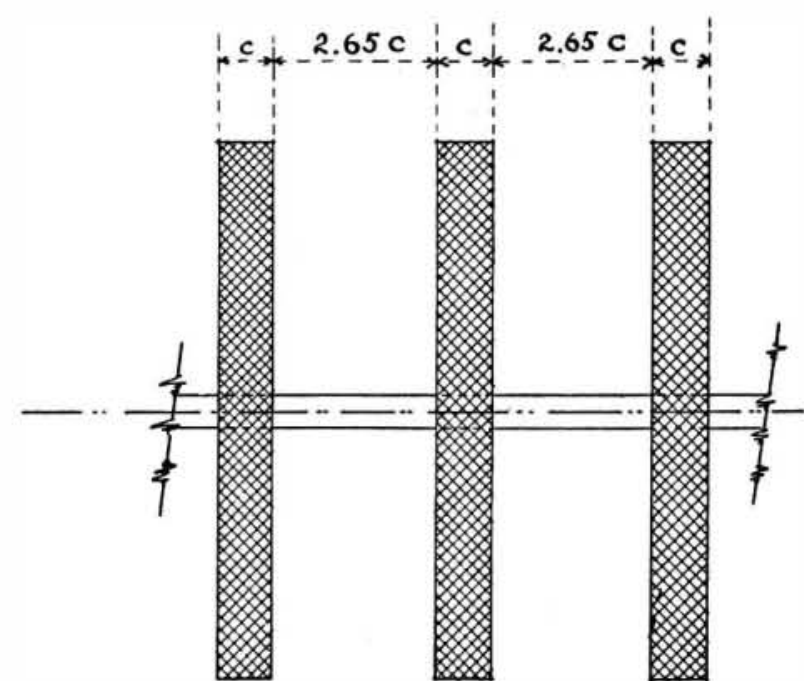


The models were excellent floaters, and could go into a dead stall and recover themselves. They could be dropped without power from a dead stall, launched with the wings making an angle of 90 degrees with the horizon, dropped sideways, dropped nose first, or launched backward and upside down, and invariably would right themselves, start to glide and make good landings.

An arrangement was tried with the two front units at an angle of three degrees and the rear unit at four degrees. This necessitatel a forwarl shifting of the two front units. The centre of gravity remained about the same, so that the distance between the centre of gravity and the centre of pressure was very much reluced, and proved that the one degree increase in angle of the rear planes had increased their lift to a considerable extent. The increase in head resistance was so small that no difference was made in the total length of flight. It was found that the negative longitudinal diedral angle was not necessary for the stability of the models.

The experiments, as far as the models wère concernel, seemed to prove in every way that the original theories were

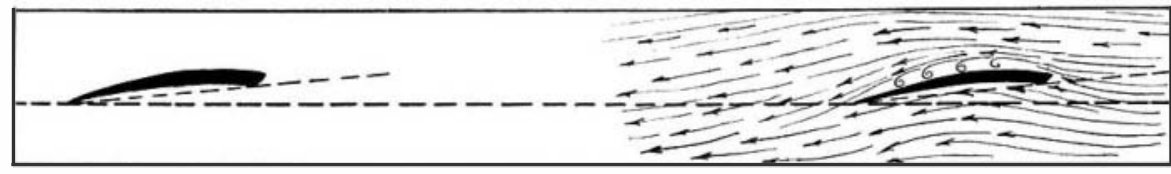

In considering full size machines with six planes similar to the models, while there is bound to be some interference of the planes, there are many mitigatin features which might be mentioned as follows :

1. The propellers will break up a large percentage of the interference between the first and second set of planes.

2. The planes may be made thinner, and consequently more efficient.

3. The aspect ratio of the individual planes can be inimrovel, as the tandem planes may be very long and narrow without increasing the total span to an unreasonable extent.

4. A system may be worled out for vertical control which will entirely eliminate the elevating rulder, thus saving considerable loss of efficiency.

5. Weight for weight the tandem machine will be great amount stronger, or if the factor of safety is the same the tandem will be lighter than the other.

6. No stabilizer, automatic or otherwise, would be required, as the tandem is in herently stable.

7. The tandem permits of larger constructions than have as yet been attempted.

\section{European Apple}

\section{Beverages}

Iv view of the health-giving properties of the apple it is regrettable that non-alcoholic apple beverages fall so far behind those prepared from the lemon in popularity. It is said of the apple that it is the most commercial of fruits and in Europe people are ac customed to value an orchard in accordance with the number of apple trees it contains. Unfortunately, however, the quality of the European ap ples is far behind the quantity; apple production in $\mathbf{E u}$ rope is so far from being profitable that it does not prevent the importation of the fruit from foreign countries, particularly from America, in great quantities. The average quality is undeniably poor and in consequence of the planting of inferior varieties and the keeping quality of the fruit is quite limited.

The methods of working up the fruit in Europe are so primitive that American dried apples are not expose to any serious competition by the home product.

The marmalade industry employs for its cheape products the refuse from ring apple production, especially parings and cores, which are imported by the shipload. Before the war even the soft drink inlustry employed dried American apple peelings for the production of apple essences.

Of somewhat more importance is the use of apples for the preparation of soft drinks. Drinks prepared from the natural fruit are naturally regarded as being more valuable than the artificially aromatized im itations. Considerable speculation has been indulge in as to whether beverages prepared from the dried
Fig. 2

ruit, especially such as are made from peelings an reparel from the fresh fruit. In favor of the drinks preparel from the dried by-products is the fact that in these the larger part of the aroma is still contained in the peelings, even after they have been subjected to drying process. Furthermore, it is a much easier and simpler matter to prepare a beverage from the drie product than from the fresh juice, which is so easily exposed to alteration by alcoholic fermentation. Furhermore, in the driel fruit a large part of the albuminoils not only make the juice difficult to clarify, but influence its keeping quality. From a practical standpoint the most prominent alvantage in favor of the driel fruit is the fact that the manufacturer is confronted only by the simple problem of redissolving the dried soluble components of the material, which he an do in such a way as to control the concentration

2

of the liquid, and in consequence is able to turn out a uniform product. To attain such a uniformity is impossible with the fresh juice on account of its rarying composition and particularly its raried acid content, on which chiefly depends the flavor of the beverage. As a matter of fact, the natural acil content of apple juice is from 0.5 to 0.9 per cent. too low for the production of the desired palate-fullness. To attain this palatefullness about $1.5 \%$ of acil is necessary. A juice with less than this amount of acil is likely to appear to the arerage palate as lacking zest. The beverages prepared from the dried apples contain the natural extractive matters of the fruit, such as the fruit sugar, the fruity acids and the nutritive salts, of which the phosphoric acid compounds are the most valuable. Since in the production of a drink with the desired acil content the dilution of the re-lissolved substances is not carried as far as is the case with the natural$$
\text { 邽 }
$$
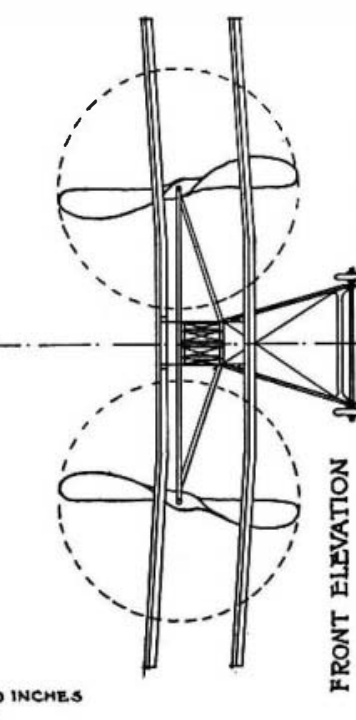
times. The first vessel is now removed from the system and its place is taken by the second one, while the reserve vessel which has just been filled with fresh material is connected with the vesse which has just yielded 400 liters extract. In this way a fresh vessel is brought into operation and 400 liters of juice are obtained every two hours. During the manipulation the hot water becomes considerably cooled, and reaches the last vessel after it has lost a great deal of its heat. The last extractions are, therefore, cold extractions, which is of great importance for the taste of the product. This method, therefore, is an improvement on the one previously employed, as it eliminates entirely the cooked taste. To eliminate such albuminoids as it now contains, the extract is heated to $80^{\circ} \mathrm{C}$. After cooling it is filtered clear through a vacuum filter and carbonated in a mixing kettle under a pressure of $1 \frac{112}{2}$ atmospheres (22 lbs. per square inch) and then bottled and closed with the well-known crown corks. The process of sterilization finishes the manufacture.

The finished average contains about $1.5 \%$ of total acid, $20 \%$ of natural fruit sugar and $0.8 \%$ of mineral matters, of which the phosphoric acid compounds, amounting to valuable.-Pure Products.

\section{Polishing Glass}

WHEN acids are used for polishing glass instead of the customary mechanical methods with abrasive materials, the irregularities in the surface are removed by solution of the glass. Hydrofluoric acid is chiefly used, but other acids (such as sulphuric acid) purposes such as the solution of calcium fluoride formed by the action of the hydrofluoric acid. A solution suitable for polishing Bohemian lead glass consists of water 1 part, sulphuric acid 2 parts, and hydrofluoric acid 1 part; for other glasses the most suitable solution should be found by

$\overline{\text { Fig. }} \mathbf{3}$

juice, the dried apple beverages contain more sugar and other components in proportion to liquid, and therefore are more nutritive and satisfying than the juice in its original condition.

The process of manufacture requires but few mechanical appliances, since the process is merely a thorough extraction with hot water. For this purpose there are employed five cylindrical enameled vessels, each with a capacity of 600 liters. In each of these ressels there is a false bottom covered with a straining cloth. Each is provided with a tight enameled cover, and to facilitate removing of the exhausted material is provided with a tipping device. These extractors must communicate with one another; the necessary connections are made through easily removable enameled, or better, aluminum pipes and the extractors are provided with draining cocks of the same material. Four of these vessels are continually in operation, while the fifth is cut out of the series for the purpose of 政 